\title{
RÉFLEXION SUR LE RÔLE HYDROLOGIQUE DES AMÉNAGEMENTS ANCIENS DES VERSANTS ET DES TALWEGS : L'EXEMPLE DES CÉVENNES
}

\author{
Claude MARTIN, Jean-François DIDON-LESCOT et Joël JOLIVET
}

UMR 6012 “ESPACE”, Département de Géographie, 98 Boulevard Édouard Herriot, BP 3209, 06204 NICE Cedex 03. Courriel : martincl@infonie.fr

Résumé : Les anciennes terrasses de culture, aujourd'hui colonisées par la forêt, n'ont plus de rôle hydrologique notable. Les nombreux ouvrages qui barrent les talwegs (tancats), construits pour lutter contre l'érosion, sont susceptibles de réguler les débits d'étiage. Mais, sans coupe de la ripisylve, leur impact apparaît essentiellement négatif.

Mots-clés : bassins versants, mesures hydrologiques, étiages, terrasses de culture, tancats.

Resumen : Las antiguas terrazas de cultivo, hoy día colonizadas por el bosque, no tienen ningún papel específicamente hidrológico. Los numerosos diques que cortan los barrancos (tancats), construidos para luchar en contra de la erosión, pueden regular los caudales en estiaje. Pero su impacto aparece esencialmente negativo sin un corte previo del bosque ribereño.

Palabras claves : cuencas de drenaje, medidas hidrológicas, estiajes, terrazas de cultivo, tancats.

\section{Introduction}

Les Cévennes, dans la partie sud-orientale du Massif Central français, conservent de nombreux aménagements anciens en pierre sèche : terrasses de culture ou simples murets sous châtaigneraie (traversiers) sur les versants, barrages pour l'arrosage (paissières) ou pour lutter contre l'érosion (rascasses ou tancats) sur les talwegs.

Toutefois la déprise rurale qui a frappé cette région à partir de la fin du XIX ${ }^{\text {ème }}$ siècle s'est accompagnée du recul des activités agro-sylvo-pastorales et de l'abandon des aménagements. La forêt a envahi pratiquement tout l'espace, soit par colonisation spontanée (hêtres, chênes verts, essences du maquis), soit sous l'action de l'homme (résineux).

Des initiatives pour la remise en état de terrasses sont actuellement soutenues par le Parc national des Cévennes, à travers le développement d'activités agricoles à forte valeur ajoutée (LÉCUYER, 2006). L'intérêt patrimonial des systèmes d'aménagement anciens justifie en outre des opérations spécifiques de réhabilitation, comme celle 
menée sur les tancats de la Vallée Obscure, à l'initiative de la municipalité de Peyrolles (Gard), sous l'égide du Syndicat mixte pour l'aménagement et la gestion équilibrée (SMAGE) des Gardons, et grâce à l'implication de plusieurs partenaires, parmi lesquels l'Office national des forêts tient une place essentielle (SCHULLER et al., 2006).

Cette région, au climat méditerranéen affecté par des effets orographiques très forts, connaît des précipitations violentes en automne, lors d'épisodes qualifiés de “cévenols", au cours desquels les abats journaliers dépassent parfois $400 \mathrm{~mm}$. Le risque d'inondation constitue ici un risque majeur pour de nombreuses villes (Alès, Anduze, Sommières...), ce qui conduit les gestionnaires du milieu à s'interroger sur les effets hydrologiques des aménagements anciens lors de ces épisodes.

À l'inverse, la sécheresse estivale est responsable d'étiages sévères, ce qui a été tout particulièrement le cas au cours des dernières années. La déficience des écoulements en été représente un risque hydrologique non négligeable dans une région où l'économie repose pour une bonne part sur le tourisme. Dans ce domaine, le rôle des tancats est intuitivement perçu comme bénéfique.

En association avec les travaux lancés par l'Observatoire hydro-météorologique méditerranéen Cévennes-Vivarais du CNRS, concernant les relations entre les précipitations et les écoulements sur le bassin versant du Gardon d'Anduze (OHM-CV, site internet), l'UMR “ESPACE” conduit des observations susceptibles d'aider à mettre en évidence les liens éventuels entre les aménagements anciens et les fonctionnements hydrologiques.

\section{I - Matériels et méthodes}

\section{A - Le terrain d'étude}

Les recherches sont concentrées sur deux petits bassins versants appartenant au bassin du Gardon de Saint-Jean (Fig. 1) : la Vallée Obscure (3,93 $\left.\mathrm{km}^{2}\right)$ et le vallon du Rouquet $\left(0,58 \mathrm{~km}^{2}\right)$.

Les deux bassins versants sont constitués de roches cristallines : granite pour le bassin du Rouquet, granite et gneiss pour celui du Valescure (Vallée Obscure). Culminant respectivement à 815 et $938 \mathrm{~m}$ d'altitude, ces deux bassins sont fortement disséqués (dénivelée de $385 \mathrm{~m}$ dans la Vallée Obscure) et présentent des versants très inclinés (pente moyenne de 56,4 \% dans la Vallée Obscure).

Le bassin versant du Rouquet est dépourvu d'aménagements anciens sur les talwegs. Les terrasses de culture et les traversiers y sont en outre peu nombreux. Les aménagements anciens sont beaucoup plus présents dans la Vallée Obscure : 0,43 $\mathrm{km}^{2}$ en terrasses de culture, $0,81 \mathrm{~km}^{2}$ en traversiers, près de 1000 tancats répertoriés à ce jour (CASTEX et al., 2006). Les terrasses et, plus encore, les traversiers sont fortement dégradés. Les tancats, construits à l'aide d'énormes blocs, sont souvent assez bien conservés, ce qui a permis leur réhabilitation le long de certains valats. Très nombreux dans le vallon des Abrits et dans celui de la Bastide, les tancats sont rares sur les bassins du Cartaou et du Valescure amont, ainsi que sur le cours aval du ruisseau de Valescure 


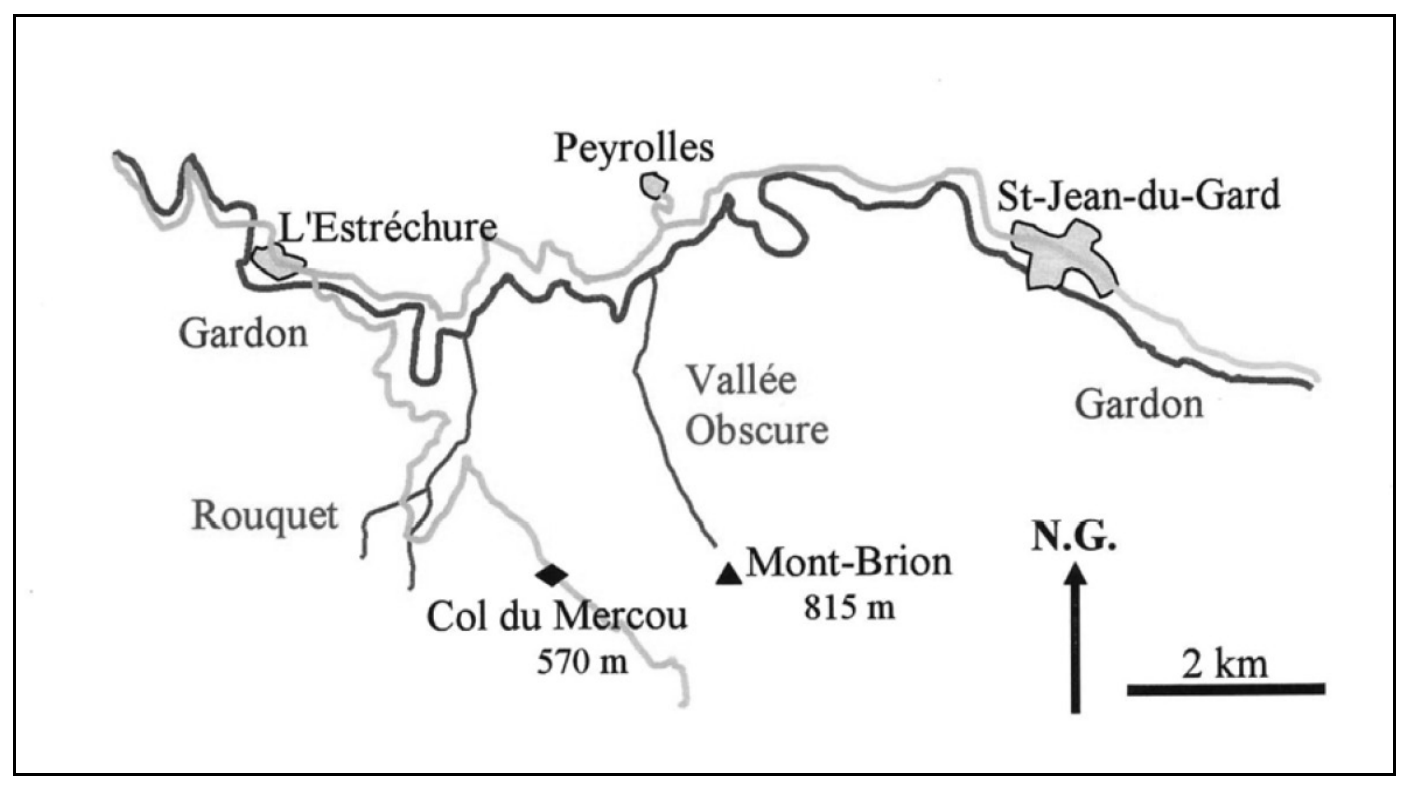

Figure 1 : Localisation du terrain d'étude.

(voir Fig. 2). Derrière ces ouvrages imposants, hauts souvent de plus de trois mètres, se sont accumulés des sédiments sur lesquels les talwegs présentent une pente faible.

\section{B - Les observations}

Le dispositif pour l'étude des phénomènes hydrologiques (Tab. 1) comporte six stations hydrométriques (Fig. 2) : cinq dans la Vallée Obscure et une dans le vallon du Rouquet. Trois stations ont une section naturelle : Valescure aval, Valescure amont, et Rouquet. La station de la Bastide a immédiatement bénéficié d'une section artificielle. Celle des Abrits a été réaménagée en juillet 2004, et celle du Cartaou en août 2005. Chaque station dispose au moins d'une échelle limnimétrique et d'une centrale d'acquisition des données associée à une sonde de pression. L'établissement des courbes de tarage est très avancé, mais les données offrent encore un caractère provisoire, en particulier pour les très hautes eaux.

\begin{tabular}{|c|c|c|c|c|c|c|}
\hline Station & $\begin{array}{c}\text { Valescure } \\
\text { aval }\end{array}$ & Abrits & Cartaou & Bastide & $\begin{array}{c}\text { Valescure } \\
\text { amont }\end{array}$ & Rouquet \\
\hline $\begin{array}{c}\text { Mise en } \\
\text { service }\end{array}$ & $\begin{array}{c}\text { août } \\
2003\end{array}$ & $\begin{array}{c}\text { février } \\
2003\end{array}$ & $\begin{array}{c}\text { février } \\
2003\end{array}$ & $\begin{array}{c}\text { mars } \\
2004\end{array}$ & $\begin{array}{c}\text { mars } \\
2005\end{array}$ & $\begin{array}{c}\text { mars } \\
2003\end{array}$ \\
\hline $\mathrm{S}\left(\mathrm{km}^{2}\right)$ & 3,93 & 0,62 & 0,52 & 0,27 & 0,93 & 0,58 \\
\hline
\end{tabular}

Tableau 1 : Stations hydrométriques de la Vallée Obscure et du bassin du Rouquet : date de mise en service et superficie contrôlée (S). 


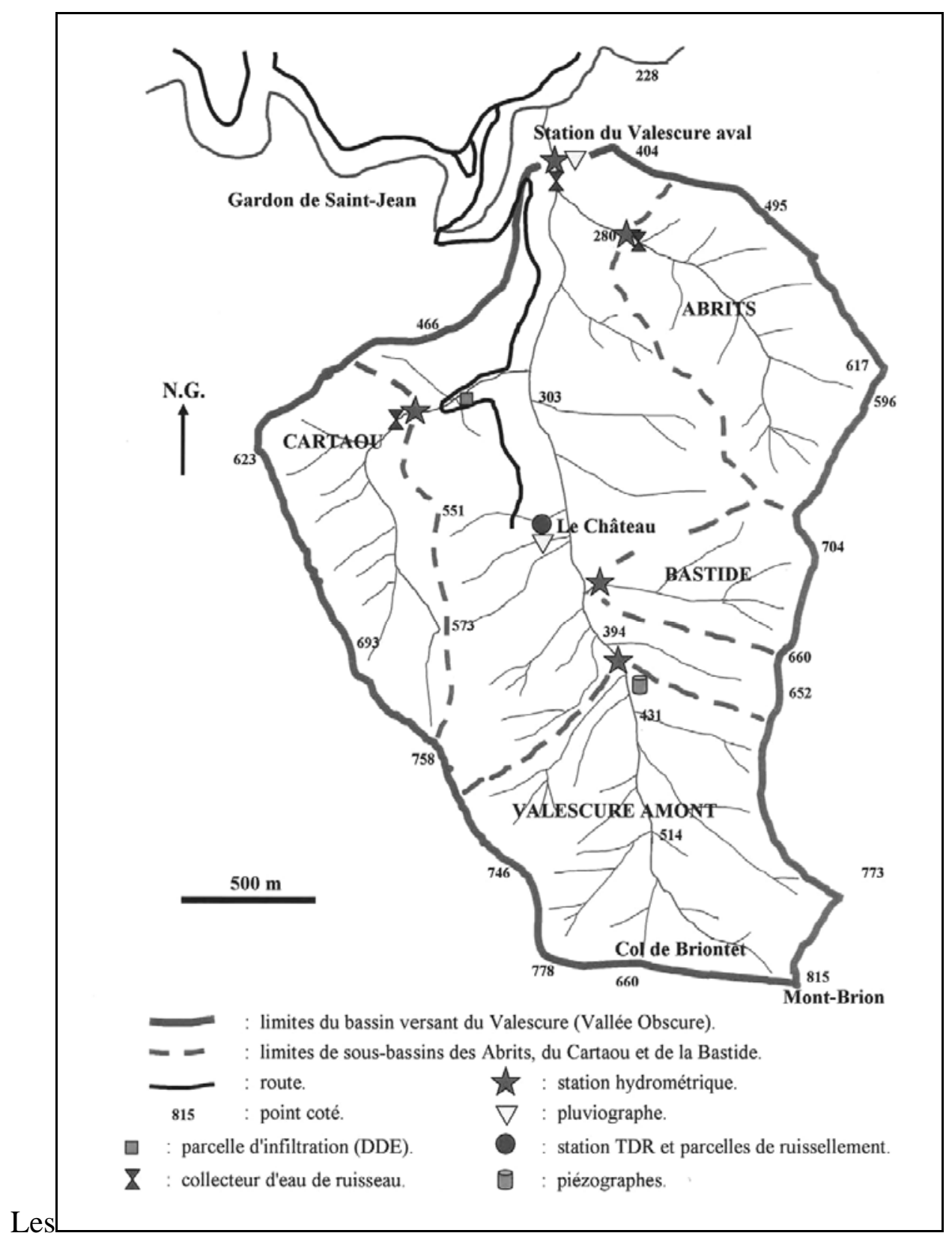

Figure 2 : Dispositif de mesure de la Vallée Obscure.

Les précipitations sont mesurées en trois points : Château de la Vallée Obscure et Valescure aval pour la Vallée Obscure, Perjurade pour le vallon du Rouquet. Les pluviographes, à double auget, d'une surface réceptrice de $400 \mathrm{~cm}^{2}$, sont reliés à une centrale d'acquisition.

En complément des suivis hydrométriques et pluviométriques, il a été effectué, d'une part, plusieurs campagnes de jaugeages en étiage sur les cours d'eau de la Vallée Obscure et, d'autre part, des traçages au chlorure de sodium sur les tancats. 


\section{II - Résultats}

\section{A - Les précipitations}

Sur la période 2003-2005, les précipitations sur la Vallée Obscure (Tab. 2) ont été supérieures de $19 \%$ à celles enregistrées par Météo-France à Saint-Jean-du-Gard (écarts de17 \% en 2003, $24 \%$ en 2004 et $15 \%$ en 2005).

\begin{tabular}{|c|c|c|c|c|}
\hline & 2003 & 2004 & 2005 & 2006 \\
\hline Année & $1710 *$ & 1476 & 1093 & - \\
\hline $01 / 05$ au 31/08 & 161 & 251 & 178 & 188 \\
\hline Pj max & 152 & 180 & 173 & 191 \\
\hline Cumul 5 Pj max & $621(38 \%)$ & $585(40 \%)$ & $447(41 \%)$ & - \\
\hline
\end{tabular}

$\mathrm{Pj}$ max : précipitations en 24 heures maximales (de 0 à $24 \mathrm{~h}$ - heures d'hiver). Cumul $5 \mathrm{Pj}$ max : somme des 5 plus fortes précipitations journalières. * : valeur en partie reconstituée.

\section{Tableau 2 : Précipitations (mm) sur le bassin versant du Valescure.}

Les précipitations annuelles moyennes à Saint-Jean-du-Gard s'établissent à 1477 mm sur 54 ans (de 1952 à 2005). Des précipitations neigeuses se produisent parfois, comme ce fut le cas en janvier 2006.

Les précipitations journalières dépassant fréquemment $100 \mathrm{~mm}$ (Fig. 3), les cumuls des cinq pluies en 24 heures les plus fortes de l'année représentent de 38 à $41 \%$ des totaux pluviométriques enregistrés sur la Vallée Obscure.

Les 8 et 9 septembre 2002, un pluviographe récemment mis en place dans la Vallée Obscure par les services du Conseil Général du Gard a enregistré $287 \mathrm{~mm}$ de précipitations en 48 heures. Toutefois les intensités les plus fortes se sont produites le 21 juin 2005, pour une pluie de $53 \mathrm{~mm}$, qui a présenté des intensités de $97 \mathrm{~mm} / \mathrm{h}$ sur 30 minutes et de $136 \mathrm{~mm} / \mathrm{h}$ sur 15 minutes, au poste du Château.

Du $1^{\mathrm{er}}$ mai au 31 août, les bassins versants reçoivent des précipitations relativement faibles. Elles l'ont été tout particulièrement en 2006. Mais la sécheresse peut se prolonger après le mois d'août. Ainsi, en 2004, 35 mm de pluie seulement sont tombés sur la Vallée Obscure du $1^{\mathrm{er}}$ septembre au 11 octobre.

\section{B - Les crues}

Sur la période d'observation, les crues les plus violentes se sont concentrées d'octobre à décembre 2003 (Fig. 3). Plusieurs épisodes assez importants ont encore été observés au printemps 2004. Le reste de la chronique présente peu de fortes crues : le 28 octobre 2004, le 31 octobre 2005 et le 29 janvier 2006. 


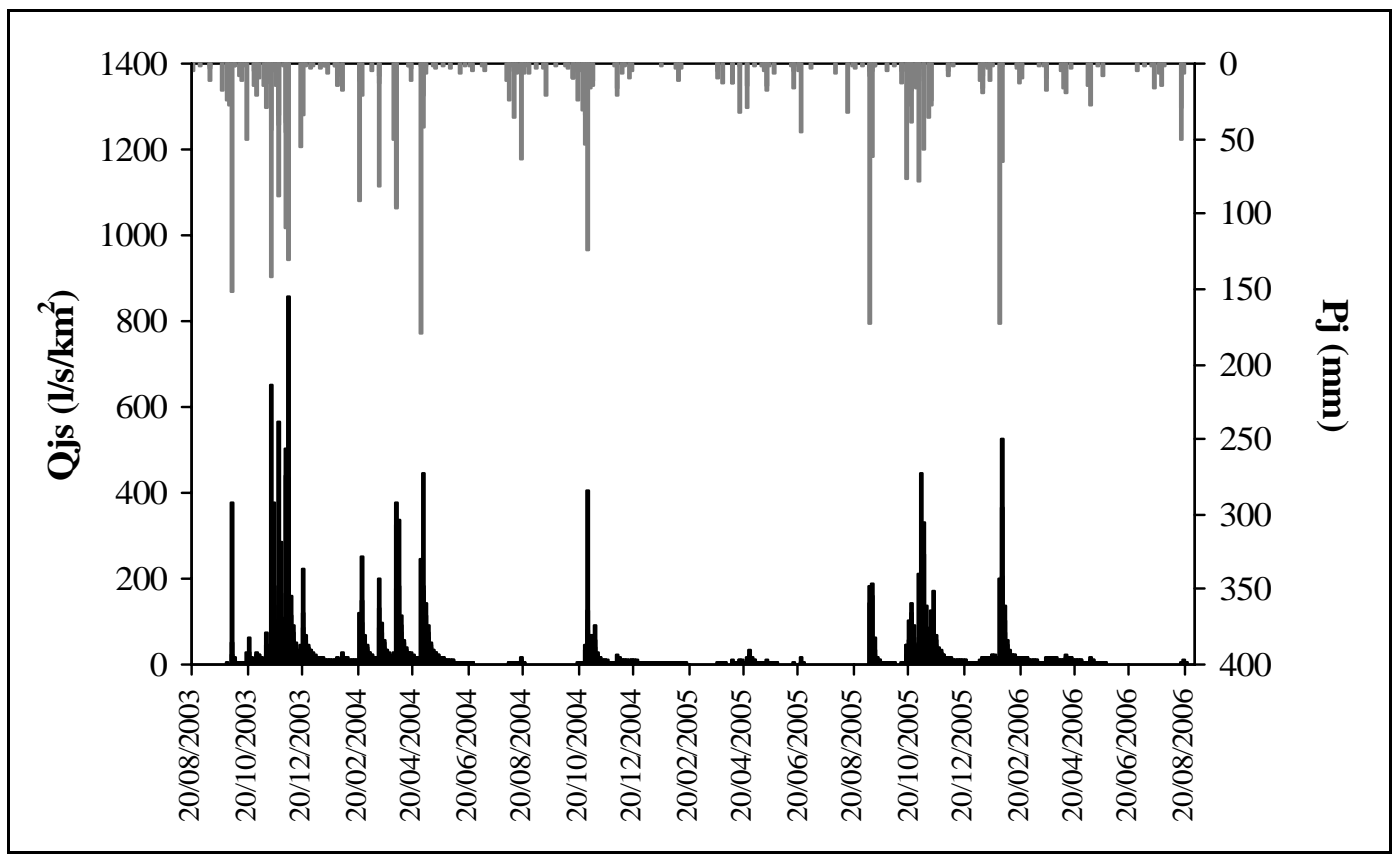

\section{Figure 3 : Débits journaliers spécifiques (Qjs) du ruisseau de Valescure aval et précipitations journalières $(\mathrm{Pj})$ sur le bassin versant.}

Sans entrer dans l'exposé de l'ensemble des résultats actuellement disponibles, soulignons la grande réactivité de tous les ruisseaux aux précipitations. Pour des précipitations données, les différences de comportement entre les bassins versants dépendent de nombreux facteurs: la présence ou non de roches ou de sols à nu, l'humidité initiale des sols et des formations superficielles, les caractères morphométriques des bassins.

Même si certains sols sur terrasses ont été “construits”, il n'a pu s'agir que de remaniements locaux sur un versant ou de transferts de matériaux dans un même bassin versant. Les systèmes de terrasses n'ont donc pas apporté un plus en matière d'épaisseur des sols. Tout au plus peut-on leur reconnaître d'avoir limité les phénomènes d'érosion dans un milieu défriché et exploité.

Dans le contexte actuel, sous couvert forestier, les aménagements de versant ne sauraient avoir d'effet notable sur les crues :

- les terrasses de culture ne sont pas localisées dans des secteurs favorables à la genèse des crues (secteurs rocheux ou aux sols peu épais, connectés au réseau hydrographique, dans le cas de pluies violentes ; zones rapidement saturées de bas de versant, dans le cas de précipitations très abondantes) ;

- les sols de la Vallée Obscure étant extrêmement perméables (MARTIN, 2006), le ruissellement par dépassement de l'infiltrabilité est généralement impossible, même pour des précipitations d'une intensité supérieure à 200 ou 300 mm/h (AYRAL, 2005). L'exploitation de parcelles expérimentales (voir Fig. 2) a montré que sur des sols soumis à l'impact des gouttes de pluie, la formation d'une croûte de battance favorise l'apparition du ruissellement (MARTIN, 2006). Envahies par la forêt, les anciennes 
terrasses de culture ne sont plus des zones contributives lors des averses de forte intensité ;

- les terrasses sont conçues pour réduire le ruissellement sur des sols nus (surfaces planes, infiltration facilitée immédiatement en arrière des murs, chenaux pour évacuer les eaux le plus vite possible vers le réseau hydrographique). Sous forêt, même après disparition d'une bonne partie des aménagements hydrauliques, les terrasses de culture ne sauraient modérer les crues liées à des précipitations très abondantes. Les suivis de l'humidité des sols par sondes TDR, réalisés sur d'anciennes terrasses, mettent du reste en évidence un ressuyage très rapide des sols (Fig. 4).

- enfin, les traversiers sont des aménagements trop sommaires pour avoir pu, un jour, tenir un autre rôle que celui de protéger les sols contre l'érosion.

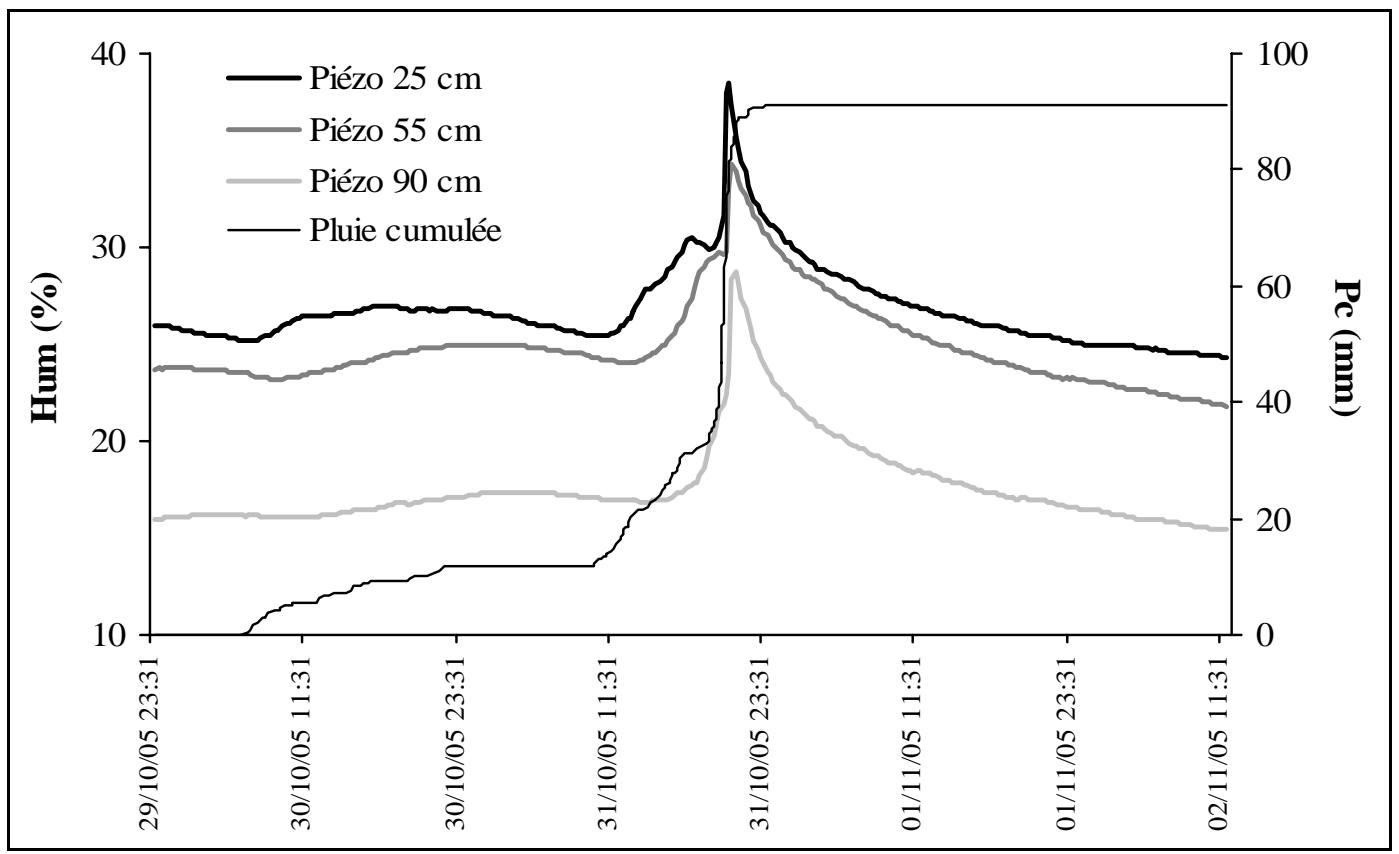

Figure 4 : Variations de l'humidité volumique des sols (Hum) sur un profil du Château de la Vallée Obscure pour l'épisode du 31 octobre 2005.

Les tancats, en revanche, exercent une influence. En remodelant le profil en long des cours d'eau en une succession de surfaces faiblement inclinées, séparées par des chutes associées à des fosses de dissipation, ils diminuent la vitesse des eaux. Mais leur finalité essentielle était certainement de limiter l'action érosive des cours d'eau : d'une part, les écoulements développant beaucoup moins d'énergie, les sapements de berge sont moins efficaces et, d'autre part, en comblant les fonds de vallon, où la pente des versants est très forte, les dépôts accumulés derrière les barrages servent d'élément stabilisateur aux terrasses construites immédiatement au-dessus des ruisseaux. La réduction des vitesses a également comme effet bénéfique de retarder les pointes de crue. Cependant l'impact sur les montées de crue reste faible au niveau des rivières principales, là où existe un risque d'inondation. En effet, les aménagements sont localisés dans de petits vallons, sur des sous-affluents, et concernent généralement moins d'un kilomètre de linéaire sur chacun d'eux. 


\section{C - Le rôle hydrologique des tancats en étiage}

L'une des raisons ayant motivé la réhabilitation d'une partie des tancats de la Vallée Obscure est l'hypothèse d'un soutien des débits d'étiage par les eaux stockées derrière ces ouvrages (site internet "Ressource en eau en Cévennes métamorphiques"). Les recherches hydrologiques qui nous ont été confiées, confirment cette hypothèse, tout en en marquant les limites.

En partant d'une première estimation réalisée par le BCEOM (2000), on peut évaluer grossièrement à $20000 \mathrm{~m}^{3}$ le volume des matériaux bloqués derrière les tancats de la Vallée Obscure. À saturation, en tenant compte des densités apparente et réelle des matériaux, la quantité d'eau contenue dans ces sédiments pourrait donc avoisiner $11000 \mathrm{~m}^{3}$. Rapportée à l'ensemble du bassin versant, cette valeur représente une lame d'eau inférieure à $3 \mathrm{~mm}$.

Des traçages au chlorure de sodium ont été réalisés sur plusieurs grands tancats alors que les écoulements se perdaient dans les sédiments : injection d'une saumure en amont de la perte et suivi conductimétrique du passage du sel en aval de l'ouvrage. La figure 5 et le tableau 3 présentent les résultats obtenus en 2006 sur un très gros ouvrage (Photo 1) situé sur le cours amont du Valescure (hauteur du tancat : 6,5 m ; longueur de la zone de dépôt : $125 \mathrm{~m}$; volume des sédiments piégés : de l'ordre de $3000 \mathrm{~m}^{3}$ ). À l'évidence, les volumes d'eau entre les points d'injection et de suivi sont nettement supérieurs à ce qu'ils seraient sans l'influence de l'ouvrage. Ainsi, pour le traçage du 31 mai, sans le tancat, le volume d'eau aurait-il certainement été bien inférieur à $20 \mathrm{~m}^{3}$, alors que l'expérimentation indique une valeur entre 144 et $492 \mathrm{~m}^{3}$.

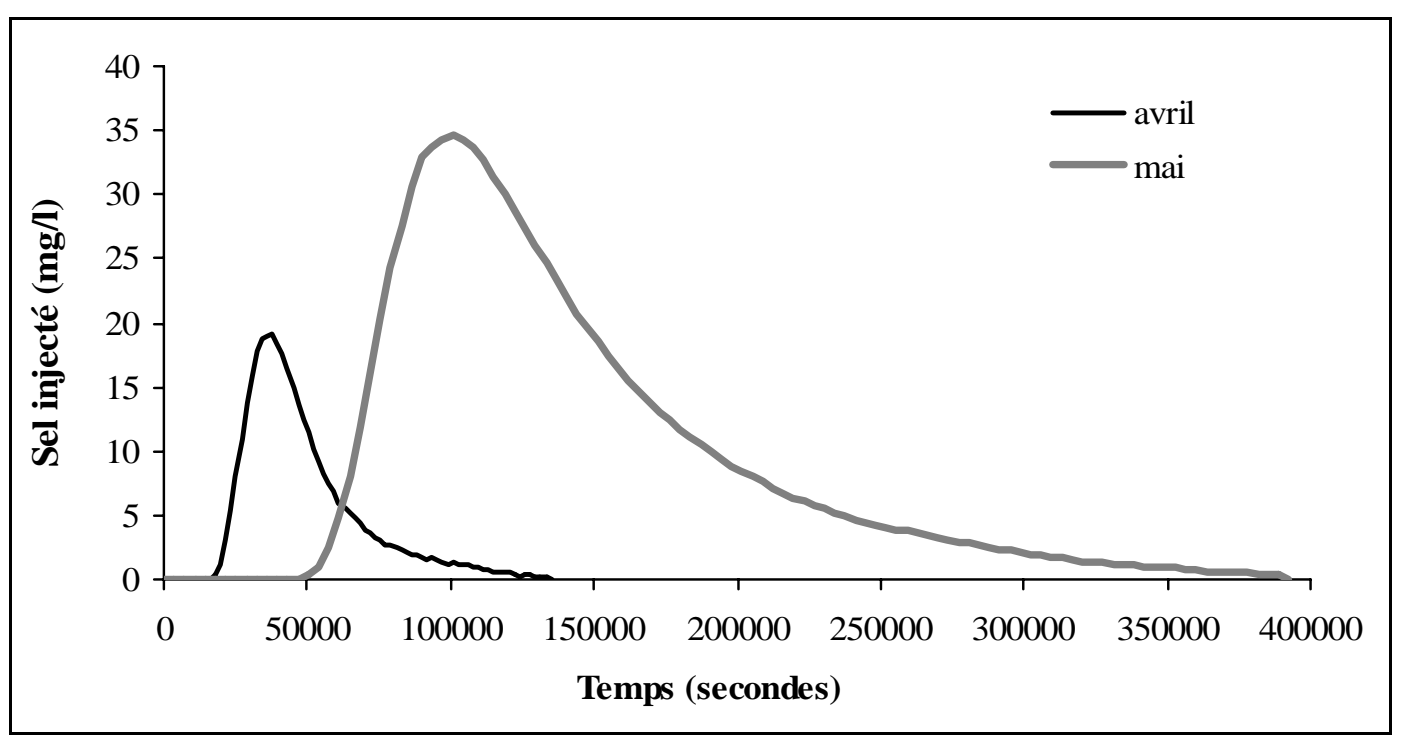

Figure 5 : Concentrations du chlorure de sodium injecté mesurées dans les eaux du Valescure amont lors des traçages d'avril et de mai 2006. 


\begin{tabular}{|l|c|c|}
\hline Date et heure d'injection & 19 avril à 12h01* & 31 mai à 09h58* \\
\hline Qté NaCl & $5000 \mathrm{~g}$ & $5000 \mathrm{~g}$ \\
\hline Distance de parcours & $135 \mathrm{~m}$ & $135 \mathrm{~m}$ \\
\hline T1 & $4 \mathrm{~h} 30$ & $13 \mathrm{~h} 00$ \\
\hline T2 & $10 \mathrm{~h} 30$ & $28 \mathrm{~h} 00$ \\
\hline T3 & $37 \mathrm{~h} 30$ & $109 \mathrm{~h} 00$ \\
\hline C max & $19,2 \mathrm{mg} / \mathrm{l}$ & $34,6 \mathrm{mg} / \mathrm{l}$ \\
\hline Qté NaCl / C & $260 \mathrm{~m}^{3}$ & $144 \mathrm{~m}^{3}$ \\
\hline Débit & $7,8 \mathrm{l} / \mathrm{s}$ & $1,4 \mathrm{l} / \mathrm{s}$ \\
\hline Vol. & $924 \mathrm{~m}^{3}$ & $492 \mathrm{~m}^{3}$ \\
\hline
\end{tabular}

*: heures d'hiver. Q $\mathrm{NaCl}$ : quantité de sel injectée. T1: temps écoulé depuis l'injection, correspondant à l'apparition de sel injecté à la base du tancat. T2 : temps écoulé depuis l'injection, correspondant à la concentration maximale du sel injecté dans les eaux. T3 : temps écoulé depuis l'injection, correspondant à la fin du passage du sel. Qté $\mathrm{NaCl} / \mathrm{C}$ : estimation par défaut du volume d'eau stocké derrière le tancat. Débit: débit moyen du Valescure amont pendant le traçage (le traçage servant de jaugeage par dilution). Vol. : volume d'eau écoulé au pied du tancat pendant le passage du sel (estimation par excès du volume d'eau stocké derrière le tancat).

\section{Tableau 3 : Données des traçages réalisés sur le Valescure amont, en avril et mai 2006.}

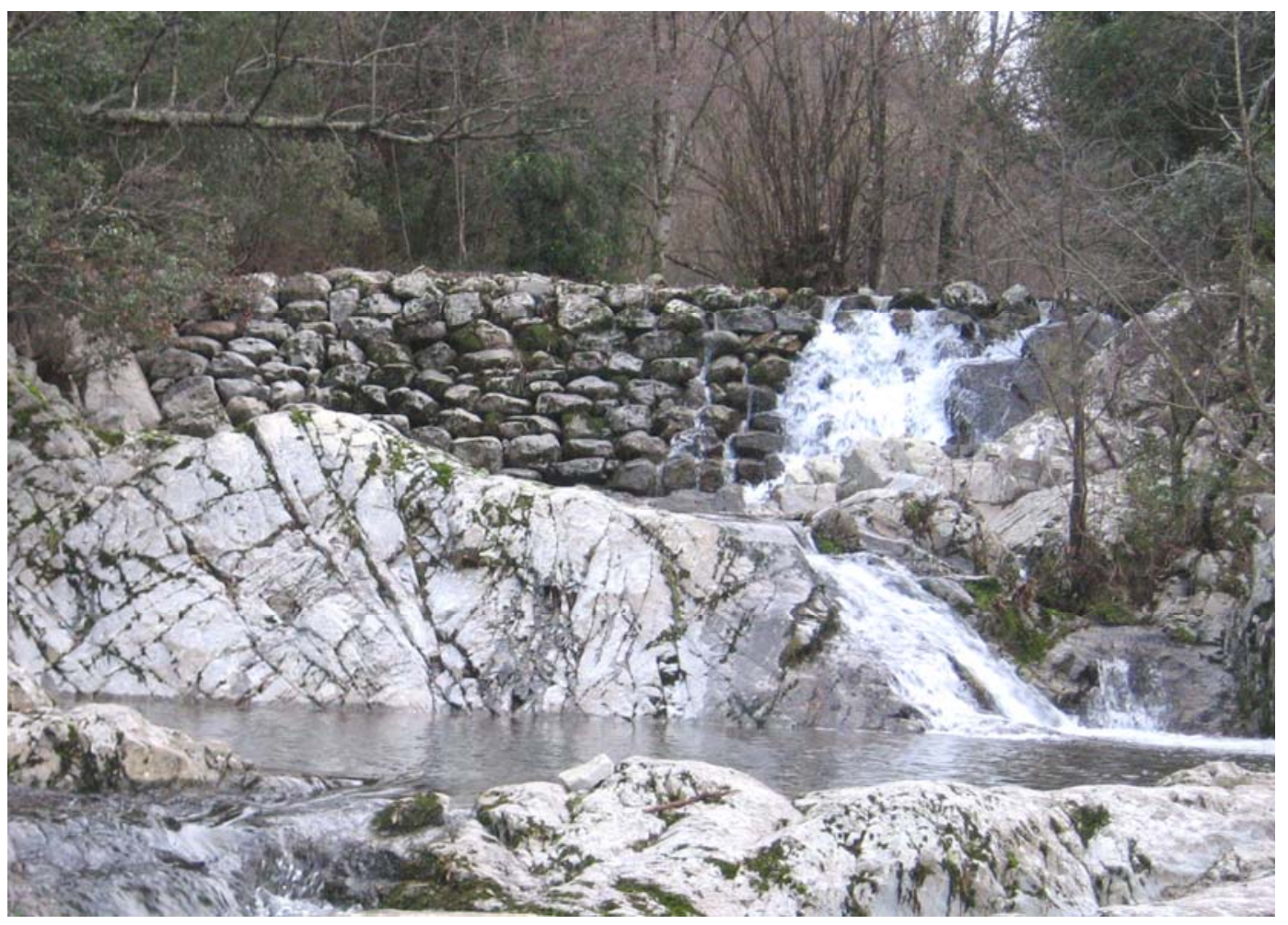

Photo 1 : Tancat dans la partie aval du Valescure (cliché C. MARTIN).

Les stations hydrométriques ont permis de mettre en évidence un effet des tancats sur les écoulements en étiage (MARTIN, 2006). Cet effet est lié aux pluies, 
généralement peu abondantes, mais assez fréquentes, qui se produisent en été. Il se traduit, en particulier à l'aval des plus grands tancats, par un retard de la réponse de l'écoulement de 24 à 48 heures par rapport aux précipitations, puis par un soutien au débit, qui devient très vite modeste, mais qui peut néanmoins se prolonger pendant une dizaine de jours. La figure 6 donne l'exemple des données recueillies en juillet 2006 à la station aval du Valescure, située immédiatement en amont d'un tancat haut de 5 mètres.

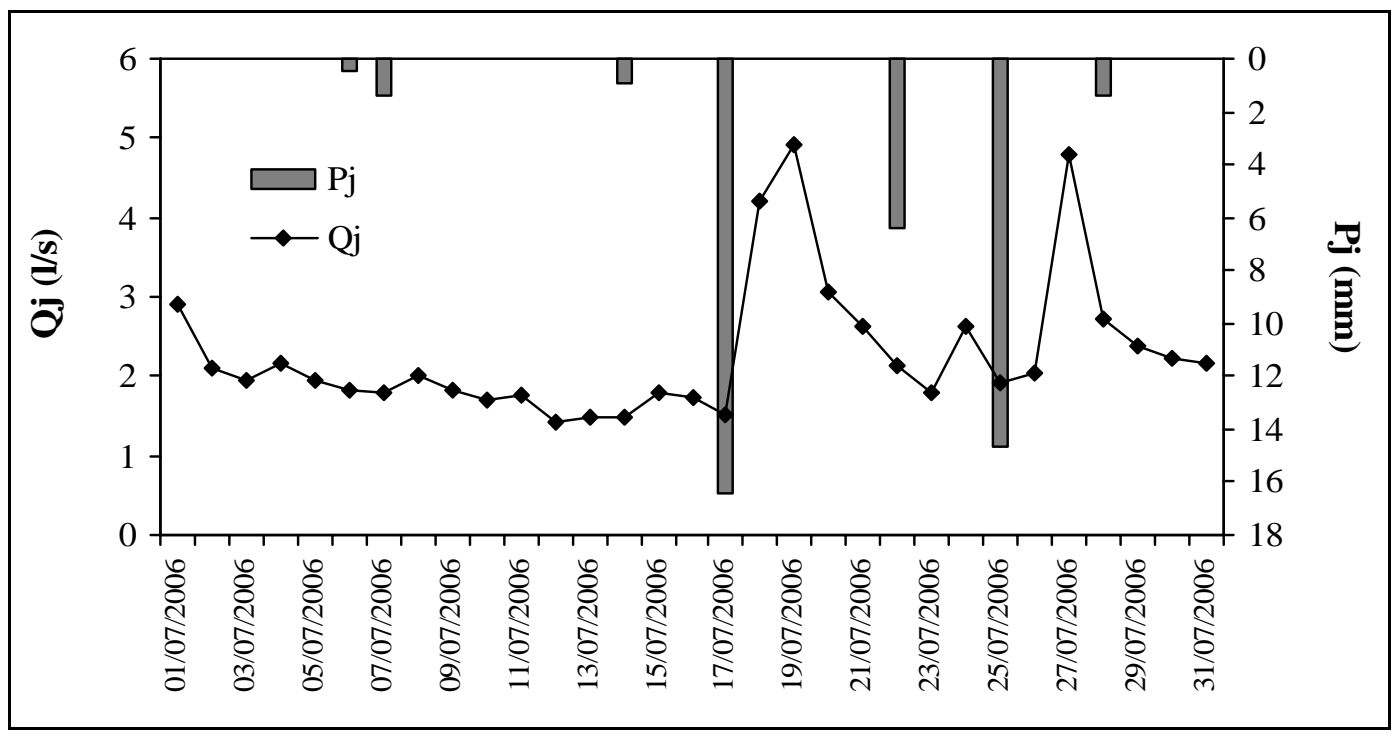

Figure 6 : Débits journaliers à la station du Valescure aval, et précipitations journalières sur le bassin versant, en juillet 2006.

Le rôle régulateur des tancats doit cependant être relativisé. En effet, la station hydrométrique du Valescure aval, qui est pourtant dominée par un très gros ouvrage, montre des fluctuations journalières des débits, liées à des pertes par évapotranspiration, identiques à celles observées sur les autres cours d'eau. Il est vrai que les dépôts en arrière des tancats sont envahis par une ripisylve souvent très dense dont l'action sur le débit est d'autant plus sensible que les eaux circulent lentement à l'intérieur des sédiments.

En fait, il semble que dans un milieu abandonné par l'homme, les tancats n'aient pas un impact réellement favorable en étiage. En dehors des périodes suivant des pluies qui ont reconstitué les stocks d'eau, les débits diminuent le plus souvent entre l'amont des dépôts et l'aval des ouvrages. Le 23 août 2005, par exemple, en milieu de matinée, à un moment de la journée où les écoulements étaient peu affectés par l'évaporation et les prélèvements par la ripisylve, le débit du Valescure passait de 0,68 l/s en amont des dépôts derrière le tancat surplombant la station hydrométrique aval (Photo 2), à 0,46 l/s au pied de l'ouvrage. De même, le 12 août 2006, en milieu d'après-midi, le débit chutait de 0,56 à $0,31 \mathrm{l} / \mathrm{s}$. 


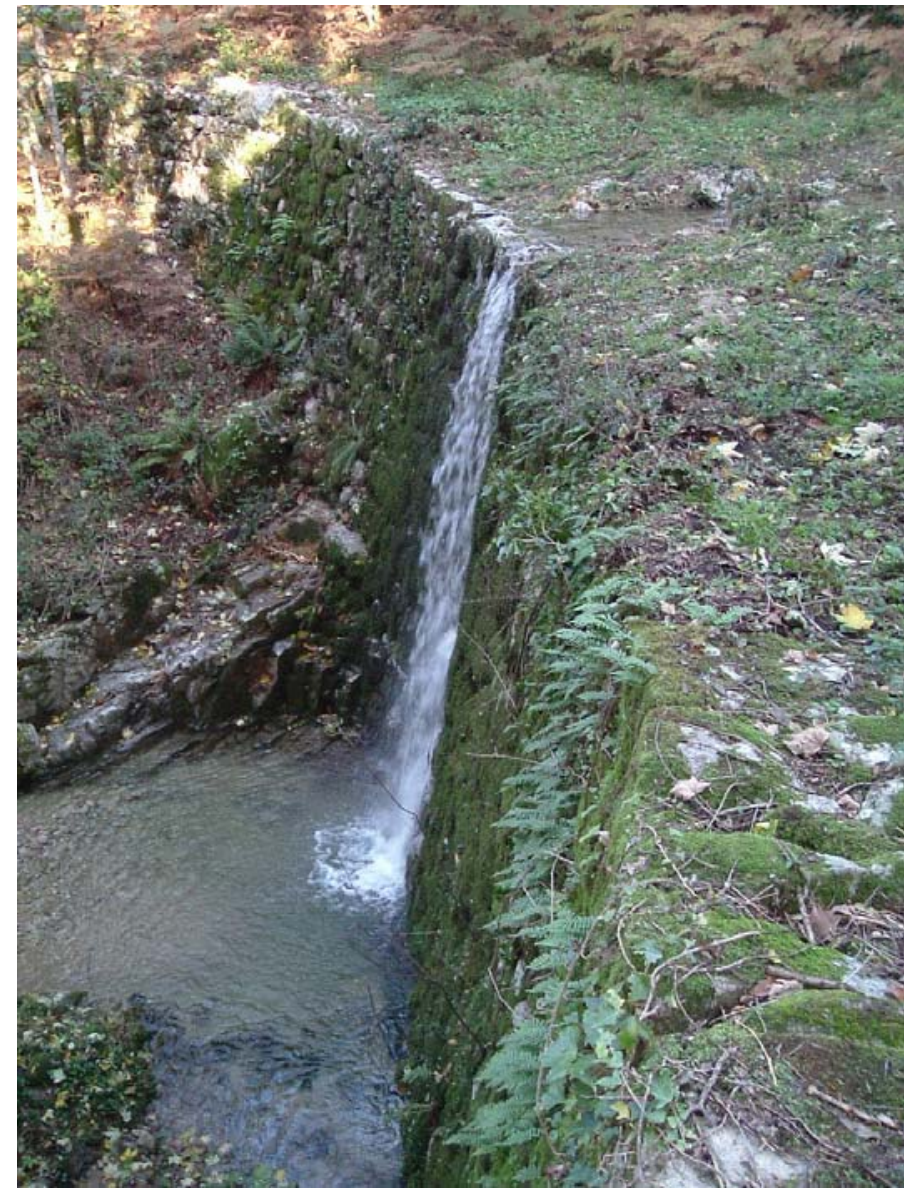

Photo 2 : Tancat dans la partie amont du Valescure (cliché J.-F. DIDON-LESCOT).

Le 26 août 2005, alors que l'étiage venait de devenir beaucoup plus sévère, la situation était inverse. En début de matinée, le débit à l'aval du tancat $(0,34 \mathrm{l} / \mathrm{s})$ dépassait déjà le débit en amont des dépôts $(0,30 \mathrm{l} / \mathrm{s})$. Avec les effets de l'évaporation et des prélèvements par la végétation, l'écart s'est accru en cours de journée : en milieu d'après-midi, les débits atteignaient respectivement 0,30 et $0,19 \mathrm{l} / \mathrm{s}$. Dans ce cas, l'écoulement à la base du tancat n'a pas été affecté par l'évapotranspiration.

\section{Conclusion}

Dans un milieu qui n'est plus exploité et dans lequel le couvert végétal s'est fermé, les anciennes terrasses de culture, pas plus que les traversiers, ne jouent actuellement de rôle hydrologique particulier. Beaucoup plus intéressant est l'impact des barrages construits en chapelet sur les talwegs pour lutter contre l'érosion (tancats). Leur localisation quasi exclusive dans de petits vallons ne permet pas d'obtenir un allongement des montées de crue des cours d'eau principaux. En revanche, ils semblent à même de soutenir les débits d'étiage en retardant l'évacuation des eaux apportées par les orages estivaux. Mais, sans la destruction de la végétation qui a envahi les dépôts derrière les ouvrages, leur impact pourrait être essentiellement négatif, car les eaux qui circulent lentement dans les sédiments, subissent de fortes pertes. Les opérations de 
forestage réalisées en 2003 et 2004 pour la restauration des ouvrages du vallon des Abrits, ont eu, du reste, pour conséquence le maintien d'un mince filet d'eau lors des étiages très sévères qui ont suivi, alors que ce ruisseau avait la réputation de s'assécher totalement presque chaque année. Les recherches sur ce point devront cependant être affinées.

\section{Remerciements}

Cet article correspond à la communication orale présentée lors de la réunion finale (Majorque, septembre 2006) du projet INTERREG III-B SUDOE “TERRISC” piloté par le Departament de Medi Ambient i Natura du Consell de Mallorca. Le travail a également bénéficié d'un fort soutien financier de la part du SMAGE des Gardons. Nous sommes reconnaissants à la Municipalité de Peyrolles, à l'Office national des forêts, à l'équipe de réhabilitation des tancats de la Vallée Obscure, au Service hydraulique du Conseil général du Gard et au Parc national des Cévennes de l'aide qu'ils nous ont apportée. Nous remercions également Pierre USSELMANN (UMR 6012 “ESPACE”) de l'aide qu'il nous a apportée.

\section{Bibliographie}

AYRAL P.A. - 2005 - "Contribution à la spatialisation du modèle opérationnel de prévision des crues éclair ALTHAÏR - Approches spatiale et expérimentale Application au bassin versant du Gardon d'Anduze.”, Thèse de Doctorat, Université de Provence, Aix-Marseille I, 310 p.

BCEOM - 2000 - "Patrimoine hydraulique du bassin de la Vallée Obscure. Maîtrise traditionnelle des eaux dans les Cévennes .”, Rapport BCEOM, 71 p + annexes.

CASTEX J.-M., MARTIN C. et ALLIGNOL F. - 2006 - "Les aménagements anciens dans la Vallée Obscure et le vallon du Rouquet.", in: "Les systèmes de terrasses cévenols. Exemples de la Vallée Obscure et du vallon du Rouquet”, Supplément au $\mathrm{n}^{\circ}$ XXXIII des Études de Géographie Physique (projet INTERREG III-B SUDOE “TERRISC"), p. 119-133.

LÉCUYER D. - 2006 - "Le rôle du Parc national des Cévennes dans la revalorisation des savoir-faire relatifs à la pierre sèche.", in: "Les systèmes de terrasses cévenols. Exemples de la Vallée Obscure et du vallon du Rouquet”, Supplément au n ${ }^{\circ}$ XXXIII des Études de Géographie Physique (projet INTERREG III-B SUDOE “TERRISC”), p. 37-44.

MARTIN C., sous la direction de - 2006 - "Espaces en terrasses et prévention de risques naturels en Cévennes.”, Projet INTERREG III-B SUDOE “TERRISC”, édit. UMR 6012 “ESPACE”, Montpellier, 32 p.

OHM-CV - (site internet) - www.lthe.hmg.inpg.fr/OHM-CV/index.php . 
Ressource en eau en Cévennes métamorphiques - (site internet) www.eau.cevennes.org .

SCHULLER F., GOMEZ N., GEORGES L., ROQUES G. et BARRÉ R. - 2006 "Le projet "Ressource en eau" : les efforts pour la réhabilitation des tancats.", in : "Les systèmes de terrasses cévenols. Exemples de la Vallée Obscure et du vallon du Rouquet”, Supplément au $\mathrm{n}^{\circ}$ XXXIII des Études de Géographie Physique (projet INTERREG III-B SUDOE “TERRISC”), p. 143-153. 